\title{
Les influences des nouvelles technologies de communication sur les organisations locales
}

Alain Laramée

\section{(2) OpenEdition}

1 Journals

Édition électronique

URL : http://journals.openedition.org/communicationorganisation/1552

DOI : 10.4000/communicationorganisation. 1552

ISSN : $1775-3546$

Éditeur

Presses universitaires de Bordeaux

Édition imprimée

Date de publication : 1 mai 1992

ISSN : 1168-5549

Référence électronique

Alain Laramée, "Les influences des nouvelles technologies de communication sur les organisations locales ", Communication et organisation [En ligne], 1 | 1992, mis en ligne le 26 mars 2012, consulté le

19 avril 2019. URL : http://journals.openedition.org/communicationorganisation/1552 ; DOI :

10.4000/communicationorganisation. 1552

Ce document a été généré automatiquement le 19 avril 2019

(c) Presses universitaires de Bordeaux 


\title{
Les influences des nouvelles technologies de communication sur les organisations locales
}

\author{
Alain Laramée
}

\section{Introduction}

1 Dans la partie québécoise du colloque organisé en mai 1991 par l'ISIC-Bordeaux 3 et le Département d'Information et Communication de l'Université Laval et consacré à "Communication et sentiment d'appartenance dans les villes des grandes agglomérations, les conférenciers ont fait ressortir certains facteurs communicationnels qui peuvent influencer le sentiment d'appartenance urbain. Ainsi, on a souligné les difficultés que présente le développement du sentiment d'appartenance dans une grande agglomération. Du fait de la taille de ces agglomérations le sentiment d'appartenance est d'autant plus difficile à développer et à maintenir que la bureaucratie et la technocratie se placent entre les élus et les citoyens. Le sentiment d'appartenance allant de pair avec la possibilité d'implication et de participation des citoyens, il ne sert à rien d'instaurer une panoplie de moyens techniques ou technologiques pour développer ce sentiment si les citoyens n'ont pas la possibilité de participer activement à la gestion de la chose publique. Par ailleurs, ces technologies peuvent, à certaines conditions, contribuer à la valorisation des dynamismes locaux.

2 L'objet de mon propos consiste donc à mettre en évidence les effets spéciaux des NTIC (nouvelles technologies de l'information et de la communication), les conséquences de ces effets sur les collectivités locales et sur leur représentation, et, enfin, proposer certaines avenues de réflexion et d'intervention.

3 Précisons d'entrée de jeu, que le sentiment d'appartenance à un groupe, peu importe sa taille, repose sur des activités d'échange et de partage de biens et de services se déroulant dans un espace commun, que l'on appelle territoire. Ces activités sont rendues possibles 
par le biais d'échanges et de partages de signes et de symboles, c'est-à-dire par la communication. Comme le rappellait récemment le géographe Henry Bakis, « le territoire diffère de l'espace géographique, car il est marqué par la société qui l'habite ». Il est une proportion d'espace humanisée. C'est le territoire qui est la référence physique de l'existence d'un groupe social et le lieu d'appartenance. À ce titre toute revendication nationale prend toujours tôt ou tard la forme d'une revendication territoriale. On comprendra donc que les communications et les télécommunications, repoussant les limites physiques de la distance entre les acteurs sociaux, jouent un rôle essentiel dans l'organisation, la structuration interne de ces territoires, dans leurs relations, les uns avec les autres, à différentes échelles, locales, régionales, nationales et internationales et enfin, affectent les représentations que ces mêmes acteurs se font de leur territoire.

Les stratégies de développement des communications, autant au plan des programmes, des techniques que des technologies, vont favoriser telle ou telle échelle et telle ou telle composante du territoire. Voyons donc, à la lumière des récentes recherches, les impacts territoriaux du développement et de l'utilisation des NTIC. Commençons d'abord par les représentations dominantes des discours théoriques, politiques et marchands.

\section{Les représentations}

5 À la fin des années 1970 et jusqu'au milieu des années 1980, les promoteurs des nouvelles technologies de communication, tant des entreprises privées, des institutions universitaires, que des organismes gouvernementaux, voyaient dans ces nouveaux moyens de communication, l'opportunité d'une nouvelle ère de convivialité où les réseaux locaux se substitueraient aux réseaux nationaux. Qu'on se rappelle les ouvrages nord américains de Marshall MacLuhan, Elvin Tofler, Merylin Ferguson, Daniel Bell, Kimon Valskis et européens de Simon Nora et Alain Mine, de Jean-Jacques ServanSchreiber, du Club de Rome, de l'Unesco et plus récemment du rapport Ribou.

Cet engouement, pour ne pas dire cette euphorie technologique, fonctionnait de pair avec la mode idéologique de l'époque qui prônait le "small is beautifull ", c'est-à-dire un certain repli sur le «local ». Les technologies de communication devenaient à la fois un nouveau secteur de développement économique, une possibilité inconditionnelle d'augmentation de la productivité, une nouvelle manière plus rationnelle d'organiser le travail, mais surtout elles présentaient des opportunités théoriques, voire "mythologiques», de stimuler la décentralisation politico-administrative et l'auto développement des collectivités locales.

7 Selon ces prospectivistes, ces nouvelles technologies, surtout celles qui intègrent la micro-informatique, les télécommunications et les banques de données, on pouvait enfin se permettre de déconcentrer les activités socio-économiques sous-jacentes à l'industrialisation en faisant émerger le « local » et le « régional » comme principal acteur de l'organisation sociale au détriment de la préséance des paliers nationaux et internationaux.

8 Ces discours étaient donc conformes à la représentation du «local» comme lieu identitaire auto-référentiel (c'est-à-dire comme espace de travail, de communication, d'échange de biens et services, en somme, le lieu par excellence d'identification et d'appartenance). Enfin, on nous promettait de résoudre le paradoxe classique opposant le local au global, en reliant le village de campagne au village mondial. 
9 Il faut dire que ce repli politique était et est encore, d'abord au plan discursif puis de plus en plus au plan pragmatique, plutôt stratégique car il s'agit de trouver une réponse aux questions que posent les différentes crises que traverse la société soit celles de l'Étatprovidence, des finances publiques, de l'énergie, de l'écologie, des nationalismes, etc.

10 Mais qu'en est-il de ces promesses et de ces espoirs à l'aube des années 1990 ? Pour répondre à cette question, nous avons inventorié les recherches récentes portant sur les influences de ces nouvelles technologies sur le terrain du développement local et régional. Pour faciliter leur présentation, nous avons regroupé ces influences sous forme de trois sphères de développement soit économique, politico-administrative et sociale.

\section{Les télécommunications au service de l'auto- développement économique.}

11 Hypothèse 1: le niveau de développement économique d'une région ou d'une collectivité est proportionnel au niveau de développement de son infrastructure télécommunicationnelle. Les télécommunications ont donc un rôle structurant et déterminant.

Certaines études ont en effet démontré la véracité de cette hypothèse à savoir que plus une région est munie de possibilités de télécommunication plus elle peut devenir attrayante pour des entreprises extérieures (Tsang, 1986). Cette possibilité de communiquer à distance diminuerait l'importance des facteurs reliés à la distance dans les choix de localisation (Planque, 1983, Wiberg, 1986) des entreprises. Toutefois selon Wiberg, l'expérience suédoise tend à démontrer que bon nombre d'autres conditions doivent être présentes pour que cet avantage serve l'autonomie régionale. Cela comprend la nécessité que les "succursales» régionales aient une grande marge de manœuvre administrative qui leur permette de constituer un réseau de contact local fort, la nécessité d'œuvrer dans un environnement très diversifié et de bénéficier d'un support des pouvoirs publics. Par ailleurs, une étude récente réalisée dans la région de Lyon (Begag, et al., 1990), tend à démontrer que « Pour qu'un équipement d'infrastructure intervienne dans le choix d'une localisation il faut en effet, soit que cet équipement ne soit disponible que dans un nombre limité de lieux, soit que son coût soit beaucoup plus avantageux en ces lieux qu'en d'autres, soit encore que son coût intervienne lourdement dans les coûts de production de l'activité économique concernée ». C'est le cas par exemple pour les centres de traitement informatique, les établissements " tête de pont " d'importants réseaux de distribution et des services administratifs de grandes entreprises œuvrant dans le secteur tertiaire.

13 Cependant, une étude américaine (Knight Gapert, 1990) portant sur les grandes cités dites «mondiales» démontre l'existence de cette corrélation du moins pour les villes de grande taille. Par exemple, cette étude rapporte que la région de New York, là où il y a le plus d'activités commerciales aux États-Unis, contient le réseau de télécommunication le plus sophistiqué et le plus étendu parmi les centres urbains. Selon cette étude, en 1988 Manhatan, à elle seule, possédait plus de deux fois les capacités de communication de la plupart des pays occidentaux, plus d'ordinateurs que le Brésil et plus de systèmes de traitement de textes que tous les pays de l'Europe combinés.

Hypothèse 2: il y a une corrélation directe entre le développement des technologies de télécommunication et l'émergence de nouvelles entreprises régionales. En permettant 
aux PME régionales d'avoir accès à l'information stratégique, jusque-là réservée aux entreprises localisées dans les grands centres urbains, les technologies de télécommunication leur permettent d'occuper de nouveaux marchés, de développer de nouveau produits (accès à la connaissance, aux brevets) et d'accroître leurs exportations extra-régionales.

La possibilité de communiquer à distance entre les entreprises locales serait génératrice de nouveaux réseaux de solidarité économique régionale. En connaissant l'offre et la demande de services et de biens sur une base régionale on serait en droit de s'attendre à voir émerger de nouvelles entreprises visant à combler la demande en région. D'un point de vue théorique, Monnoyer $(1985)$ et Lamarche $(1986,1990)$ ont développé des modèles d'application possible de cette hypothèse au secteur des services. Par contre les études empiriques de Planque $(1983,1985)$ démontrent que cette hypothèse ne peut se vérifier actuellement que pour le secteur des services reliés à l'informatique

Hypothèse 3: Les possibilités de contrôle à distance vont permettre aux grandes entreprises nationales et internationales de déconcentrer davantage leurs activités.

Plusieurs études (Planque $(1983,1986)$ Hepworth (1985), de Lesser et Gall (1987) et de Goddard et Gillepsie (1986), Bakis, 1990) ont déjà expérimenté cette hypothèse. Elles concluent que les activités exigeant une communication dialogique ont tendance à se situer dans les régions périphériques, là où les coûts de production sont plus faibles, alors que les activités exigeant une communication monologique ont tendance à se concentrer dans des grands centres décisionnels. On voit donc apparaître d'abord au plan national puis graduellement au plan international: les fonctions de décision et de recherche seront dans les grands centres (agglomérations urbanisées) et les fonctions d'exécution et de production dans les régions périphériques. Or, comme l'a démontré Planque (1990), ce sont les créations de nouvelles entreprises et de nouvelles synergies locales. Par ailleurs, l'étude de Hepworth (1985) portant sur la réorganisation du journal Globe and Mail, arrive plutôt à un constat opposé à cette hypothèse, du moins dans le secteur de l'édition. En effet, il démontre que le journal Globe and mail a pu réduire considérablement son personnel d'édition au Québec et dans d'autres provinces, en faisant éditer le journal à Toronto et l'envoyant par voie télé-informatique dans les différents points de distribution. De plus, le personnel d'administration et de gestion est presque totalement à Toronto. On pourrait citer plusieurs autres études semblables effectuées aux États-Unis, en Angleterre, en Suède, et en France, notamment celles de Henry Bakis portant sur la distribution et l'édition de la presse parisienne et sur la multinationale IBM.

\section{Les télécommunications au service de la démocratie}

Hypothèse 1 : Plus le système politico-administratif est décentralisé lors du développement d'un système d'information informatisé plus il $\mathrm{y}$ a de risque que le système d'information favorise la décentralisation.

Dans cette hypothèse, les télécommunications sont des amplificateurs de tendances existantes dans un système politico-administratif (comprenant les systèmes de gestion, de planification et de décision) plutôt que des agents de changement d'orientation. Nijkamp et Rietveld (1984) ont confirmé cette hypothèse à partir de rapports diagnostics effectués par des chercheurs de six pays : la Hollande, la Suède, la France, les États-Unis, la Tchécoslovaquie et la Finlande. Ils ont observé que plus un pays avait une orientation 
centralisatrice avant son informatisation plus la venue de la technologie avait renforcé cette orientation. L'inverse était également vrai.

Hypothèse 2: La télématique permet davantage de consultation des citoyens concernant les orientations de gestion de la collectivité.

21 En permettant une consultation plus grande la télématique deviendrait un facteur d'accroissement de la participation et de l'implication des citoyens donc de développement de la démocratie. Une étude empirique de Loiseau (1988) en France démontre que les municipalités dotées de réseaux télématiques semblent très peu portées à utiliser ce service. Une autre étude (Vitalis et Claret, 1988), aboutit au même résultat. Cette étude insiste même sur la nécessité d'un encadrement juridique, scientifique et méthodologique pour toute municipalité qui veut utiliser cette possibilité de la télématique car cette utilisation pourrait même résulter en un effet contraire, à savoir servir à la manipulation des citoyens ou de l'électorat.

Hypothèse 3: L'usage de la télématique par les citoyens leur permet d'influencer le processus décisionnel par l'accès direct aux élus et à l'information stratégique.

Tout comme pour l'hypothèse précédente évidente et malgré le peu d'études effectuées à cet effet jusqu'à maintenant, l'étude le Loiseau (1988) démontre qu'on a encore peu exploité cette possibilité et que les municipalités ne semblent pas être favorables à son développement. Il faut dire qu'il y a peu d'applications possibles développées encore dans ce domaine

\section{Les télécommunications au service des réseaux sociaux locaux}

Hypothèse 1: En permettant aux citoyens d'échanger entre eux dans un cadre hors institution, la télématique est génératrice de nouveaux réseaux de solidarité locale (au sens de Rosenvallon, 1981).

Cette hypothèse stipule que par les échanges non contrôlés, non institutionnalisés et non organisés entre les citoyens au moyen de la télématique on serait fondé de voir apparaître de nouvelles formes de regroupement et de communauté conduisant éventuellement à de nouvelles formes d'organisation sociale et au développement du sens d'appartenance et d'identité régionale. Selon Loiseau (1988), on n'a pas encore observé l'existence de tels réseaux de solidarité locale. Il faut toutefois considérer les problèmes méthodologiques de taille que présente une telle étude. Par ailleurs, ce qu'on voit apparaître de plus en plus, ce sont des réseaux déterritorialisés. C'est-à-dire des réseaux d'intérêts indépendamment des appartenances territoriales, du moins au plan local et régional : par exemple, des clubs divers, des regroupements d'association, des chercheurs, les services vidéotex (messageries diverses, bases de données,...) etc. Ces réseaux ont davantage tendance à se mouler sur l'étendue technique du réseau de télécommunication que sur les réseaux territoriaux.

Hypothèse 2 : Tout comme l'utilisation de la téléconférence dans les entreprises provoque paradoxalement des rencontres de face à face créatrices de nouvelles synergies, l'utilisation des télécommunications dans l'organisation régionale et locale peut susciter de telles rencontres créatrices de nouveaux réseaux sociaux. 
27 Afin de vérifier la véracité de cette hypothèse, Lars Quortrup (1985) a étudié le phénomène des centres d'information et de services communautaires au Danemark. Ces centres sont un compromis entre le caractère individualiste des télécommunications et la dimension émancipatoire spécifique à une relation de type face à face. Les centres servent autant à la consultation télématique professionnelle de banques de données ou à la consultation de personnes ressources situées dans des centres éloignés qu'à la consultation et à l'échange d'informations de face à face. Le fait que les acteurs sont forcés de se rendre à ces centres pour communiquer à distance crée un cadre de rencontres et d'échanges communautaires. Ces occasions de rencontres transforment ces centres en lieux synergiques d'où émergent différents projets et de nouvelles formes de liaisons sociales. Pour l'auteur, ces centres sont une sorte de réification du concept de «village global ». On trouve le même phénomène en France là où sont expérimentés les centres multimédias. Cependant, il faut noter que ce qui est un stimulant des nouvelles synergies est moins la télématique que son prétexte servant aux déplacements et aux rencontres de face à face dans ces centres.

\section{Bilan}

Que retenir de ces études et de ces résultats?

Malgré le fait que ce champ de recherche soit encore très peu développé et que, de ce fait, nous ne pouvons pas identifier de prototypes, de modèles analytiques ni d'intervention, nous pouvons observer certaines tendances lourdes.

1. Les effets spatio-économiques des nouvelles technologies de communication sur le développement local sont beaucoup plus différenciés que le voulaient leurs promoteurs. Ces effets semblent favoriser davantage une centralisation des activités de décision dans les grands centres urbains au plan national, et de plus en plus dans les grands centres mondiaux au plan international.

31 2. Les usages sociaux des télécommunications sont très peu développés et promus, et lorsqu'ils le sont, ils sont déterritorialisés, allant dans le sens de l'éclatement du territoire d'appartenance.

32 3. La télé-démocratie est encore au stade de mythe. Ce qu'on voit c'est davantage un éloignement des citoyens des centres de décision, l'apparition de ce que Friedman et Castells appellent, une nouvelle interface entre les élus et les citoyens, qu'on pourrait appeler les informocrates, une sorte de mixte d'informaticiens et de technocrates, développant et utilisant des moyens puissants d'information complexifiant davantage la relation avec les citoyens plus souvent qu'autrement démunis de culture technologique et démunis d'infrastructure télécommunicationnelle.

Ces tendances empiriques semblent donc militer dans le sens plutôt opposé aux pronostics et aux prévisions théoriques. Elles démontrent également que ces technologie doivent être considérées tout comme d'autres infrastructures c'est-à-dire qu'elles se développent et se diffusent en fonction des acteurs sociaux, des infrastructures, des intérêts et des volontés en place.

Laissé à lui-même, le développement de ces technologies n'est certes pas neutre, mais il est un facteur de valorisation des modèles et des stratégies de développement préexistants, ce qui lui confère, par le fait des investissements et des équipements qui l'accompagnent, un caractère structurant l'espace à moyen et à long terme. Or les 
économies modernes des pays industrialisés semblent privilégier le libéralisme économique dans ce domaine et la déréglementation.

Or, comme en font foi les études pré-mentionnées, la logique des « lois » de l'économie de marché, conduit « l'autoroute électronique » non pas à décentraliser mais à valoriser une dialisation spatiale de l'économie conduisant ainsi à l'appauvrissement des localités dites périphériques en ressources à valeur ajoutée.

En retour, ces effets ont marqué également les discours politiques et idéologiques. Au plan symbolique et référentiel, les échanges électroniques de données, les possibilités de surveiller et contrôler à distance, de disposer des informations de manière instantanée, d'élargir l'étendue de l'espace de marketing des entreprises ont contribué à créer le syndrome du «mondial». Les entreprises peuvent de plus en plus penser «mondial» alors que les collectivités peuvent de moins en moins penser « local ».

Cependant, ce syndrome trouve sa contrepartie locale beaucoup moins fantaisiste. Dans les grands centres urbains, ceux qui bénéficient le plus des effets économiques des NTIC, les citoyens partent de leur banlieue où ils vivent chacun de plus en plus isolé devant leur appareil de télévision de plus en plus «interactif », prennent de plus en plus de temps à se rendre dans des centre-ville de plus en plus congestionnés pour aller travailler dans des entreprises qui sont en relation télécommunicationnelle avec de plus en plus de gens, de plus en plus éloignés et de plus en plus instantanément. Voilà le paradoxe existentiel de cette tendance sauvage pour l'économie de l'information: concentrer les employés pour déconcentrer les entreprises.

38 Par ailleurs, l'idéal d'une "télédémocratie " à piton augmentant la transparence des administrations publiques et permettant au citoyen de participer davantage aux choix et aux orientations de sa société a plutôt cédé la place à une « infocratie ».

Ce qu'on retrouve, c'est une administration publique de plus en plus capable d'intégrer et de centraliser ses différents systèmes de contrôle et de surveillance des activités et des citoyens, dotée de professionnels de plus en plus spécialisés (Géomatique, SIURS, SID, S.E., S.I.A.D., S.I.G...) et nombreux agissant comme intermédiaires/experts entre (sinon audessus) des citoyens et des élus.

En somme, les velléités émancipatrices d'autonomie locale des télécommunications ne se sont pas manifestées et rien ne laisse présager actuellement un revirement de tendance. À la suite de ces transformations profondes et accélérées du « local » et en contradiction avec les promesses d'émancipation des télécommunications, d'aucuns diraient les utopies, la représentation du local, parfois mythique, comme territoire où se construisent et reconstruisent des solidarités sociales, où se théâtralise la quotidienneté, comme lieu identitaire, comme espace d'inter-subjectivité, qui servait de référentiel aux revendications et aux mesures de développement de l'autonomie du «local » a cédé la place à une représentation du «local» comme lieu hétéro-référentiel, fonctionnel, informatisé, organisé, efficace et fournisseur de biens et services.

41 Le «local» sert de porte au "global». La mode et le discours sont au "planétaire », à l'instabilité, à la turbulence, à la complexité, à la mobilité, à l'exportation, à l'expansion, à la modernité, à l'abstraction, à la ville mondiale, à l'abolition des frontières, à l'agora électronique... en bref, aux notions et aux représentations sans référence territoriale. Ces représentations supportent une rhétorique justifiant la croissance pour la croissance et véhiculent un symbolisme local orienté vers l'extérieur. 
42 Mais cette nouvelle représentation de l'autonomie sociale, issue et portée par une réduction de la communication sociale à l'utilisation spatio-fonctionnelle des télécommunications contient une grande part de mythe non pas intégrateur mais différenciateur.

43 Tôt ou tard, le manque d'ancrage territorial des stratégies de développement et d'utilisation des NTIC fera ressortir, sauvagement peut-être, de manière subversive, voire violente, ce besoin non assouvi d'auto-référence, d'appartenance, bref d'identité transgressé par ce modèle artificiel et strictement utilitaire de communication entre les humains privilégiés par les stratégies actuelles. On le voit de plus en plus d'ailleurs à travers le monde avec l'augmentation des revendications nationales, ethniques et religieuses qui prennent la forme de revendications territoriales.

\section{Conclusion}

En conséquence, si la situation est telle qu'elle est, c'est probablement dû d'abord à l'échec de nos modèles de développement urbain qui sont motivés davantage par des objectifs de croissance économique et la foi inconditionnelle au libre marché que de développement social. Les NTIC ne font qu'amplifier ce mouvement. Alors que, dans ce domaine, tous les observateurs avertis le confirment, les interventions publiques et politiques sont nécessaires si on veut éviter un développement sauvage, chaotique et laissant des travers irréversibles sur l'organisation locale. Le sentiment d'appartenance s'accommode mal de la logique économique et de l'imaginaire qui l'accompagne.

À ce titre, les collectivités locales n'ont pas encore effectué la transformation de représentation nécessaire pour contrer les effets pragmatiques, souvent invisibles, parfois souterrains, de cette représentation «mondialisante » et standardisante au plan idéologique mais déterritorialisante et différenciante dans la pratique.

En conséquence, les collectivités locales ont une responsabilité et un défi important à relever ; celui de refuser d'être banalisées, de n'être considérées que comme des éléments du système de flux communicationnel qui traverse l'espace pour se redéfinir en tant qu'entité et territoire intégrant les différents réseaux de communication plutôt qu'étant intégrés par eux et adoptant un rôle proactif dans leur développement et leur diffusion. L'urgence est d'autant plus grande que les états-nations occidentaux et les grandes cités «mondiales » semblent avoir déjà défini leur cadre de référence en optant pour le global au détriment du local (libre-échange avec Canada/USA/Mexique, mondialisation de l'économie, déréglementation, Europe de 1993, libéralisation accentuée des pays de l'Est, désengagement de l'État, normalisation technologique...).

Pour les collectivités locales, ce défi passe donc par :

- 1. Une connaissance accrue des dynamismes spatiaux des NTIC sur leur territoire. À ce titre, la recherche universitaire, quoique plus développée en France qu'au Québec dans ce domaine, a un rôle accru et majeur à jouer. La recherche empirique, notamment les études de cas sont encore embryonnaires et les études comparées me semblent souhaitables pour la reconstruction théorique en ce domaine.

- 2. Une évaluation et une maîtrise sociale de l'utilisation des NTIC en fonction des orientations de développement. Là encore la recherche universitaire doit être accrue surtout au plan des instruments méthodologiques. 
- 3. Une reconstruction de l'image identitaire de la collectivité locale intégrant les nouveaux réseaux techniques aux réseaux de communication traditionnels. Accentuation de la dimension auto-référentielle par rapport à l'hétéro-référentielle.

- 4. L'élaboration de programmes, de politiques et de stratégies valorisant l'appropriation locale de ces nouveaux réseaux.

- 5. Une présence proactive dans la règlementation et le développement de ces nouvelles technologies.

- Voilà, il nous semble, autant de défis que de conditions pour que les NTIC servent l'autodéveloppement local et non l'auto-asservissement global.

\section{RÉSUMÉS}

Alors que se développe un discours administratif sur le bien-fondé de la prise en charge des collectivités locales de leur propre destin, qu'on parle de régionalisation et de déconcentration, les télécommunications tendent à abolir les frontières, à augmenter les possibilités de contrôle à distance et à standardiser les avenues de développement. Ce contexte pose un défi majeur à l'identité des collectivités locales.

\section{AUTEUR}

\section{ALAIN LARAMÉE}

Alain Laramée, PH.D, professeur en communication organisationnelle, dirige le module Communciation de la Télé-université du québec. Il est l'auteur de quatre livres, de nombreuses conférences et publications sur la recherche, la planification et la gestion de la communication dans les organisations. 